\title{
Quantum computing based on semiconductor nanowires
}

\author{
Sergey M. Frolov, Sébastien R. Plissard, Stevan Nadj-Perge, \\ Leo P. Kouwenhoven, and Erik P.A.M. Bakkers
}

\begin{abstract}
A quantum computer will have computational power beyond that of conventional computers, which can be exploited for solving important and complex problems, such as predicting the conformations of large biological molecules. Materials play a major role in this emerging technology, as they can enable sophisticated operations, such as control over single degrees of freedom and their quantum states, as well as preservation and coherent transfer of these states between distant nodes. Here we assess the potential of semiconductor nanowires grown from the bottom-up as a materials platform for a quantum computer. We review recent experiments in which small bandgap nanowires are used to manipulate single spins in quantum dots and experiments on Majorana fermions, which are quasiparticles relevant for topological quantum computing.
\end{abstract}

\section{Introduction}

The basic element of a quantum computer is a quantum bit, or qubit, which in analogy to a classical bit contains information. A quantum degree of freedom can be charge, spin, photon polarization, and magnetic flux. Several quantum systems are being explored as qubits, each with their specific advantages and challenges: single atoms in ion traps (see the article by Hite et al. in this issue), NV defect centers in diamond (see the article by Gordon et al. in this issue), and superconducting circuits (see the article by Oliver and Welander in this issue). Among these, semiconductor-based qubits are attractive due to their electrical tunability and ease of integration with the electronics industry. However, the search for a perfect semiconductor platform that simultaneously satisfies the requirements of fast quantum control, long coherence time, and scalability to thousands of coupled qubits continues.

A prominent semiconductor system in which single and double qubit operations were demonstrated is a two-dimensional electron gas (2DEG) at the interface between GaAs and AlGaAs. ${ }^{1}$ By using metallic gates on top of the heterostructure to isolate small regions of $2 \mathrm{DEG}$, quantum dots containing single electrons have been electrostatically defined. Rather than following the complementary metal oxide semiconductor route and using charge as an information carrier, electron spin is used in these quantum dot qubits for carrying information. ${ }^{2,3}$
This is because charge noise in semiconductors does not allow quantum states of charge to survive much longer than a nanosecond. ${ }^{4}$ While two-dimensional systems currently lead the race among semiconductor qubits, they are still a long way to a practical quantum computer. Among challenges going forward is the need to simultaneously carve zero-dimensional quantum dots out of a 2D sheet of electrons, and couple thousands of these dots while only being able to place control electrodes on top of a heterostructure. ${ }^{5}$ The need to increase spin coherence times may require changing the materials that host the quantum dots. ${ }^{6}$ Here, a drawback of two-dimensional systems is the limited design freedom of the material. To avoid strain and consequent incorporation of dislocations, highquality 2DEGs can only be fabricated with (nearly) latticematched materials, which is possible only for a small set of material combinations.

A new solid-state platform that has recently demonstrated promise for quantum computing is semiconducting nanowires. Nanowire qubits yield the fastest electrical spin manipulation times to date for single spins in quantum dots. ${ }^{7}$ In addition, the first signatures of novel Majorana fermion quasiparticles, which are their own antiparticles and represent the building blocks of topological qubits, were obtained in nanowires. ${ }^{8}$ This progress was possible because nanowires allow for almost unlimited material design freedom in terms of chemical

Sergey M. Frolov, Department of Physics and Astronomy, University of Pittsburgh; frolovsm@pitt.edu

Sébastien R. Plissard, Technische Universiteit Eindhoven, Department of Applied Physics, The Netherlands; s.r.plissard@tue.nl

Stevan Nadj-Perge, Department of Physics, Princeton University; snadj@princeton.edu

Leo P. Kouwenhoven, Kavli Institute of Nanoscience, Delft University of Technology, The Netherlands; I.p.kouwenhoven@tudelft.n

Erik P.A.M. Bakkers, Technische Universiteit Eindhoven, Department of Applied Physics, The Netherlands; e.p.a.m.bakkers@tue.nl

DOI: $10.1557 / \mathrm{mrs} .2013 .205$ 
composition, stacking, and geometry. Due to the small dimensions, strain can be relieved at the surface, and nanowire heterostructures of Group IV (e.g., Si/Ge), ${ }^{9}$ Group III-V (such as $\left.\mathrm{InP} / \mathrm{InAs},{ }^{10} \mathrm{GaAs} / \mathrm{GaP},{ }^{11} \mathrm{GaN} / \mathrm{AlGaN}^{12}\right)$, and Group II-VI elements $(\mathrm{ZnSe} / \mathrm{ZnTe})^{13}$ have been demonstrated. More recently, combinations of different classes of semiconductors, such as Group IV and Group III-V, have also been realized within a single nanowire maintaining high material quality. ${ }^{14}$

One advantage of nanowires from the qubit scalability point of view is that they allow for multiple local metallic or superconducting contacts and electrostatic gates on top, beneath, and next to the wire. ${ }^{15}$ For example, a chain of quantum dots defined by bottom gates can host qubits in a single wire, while adjacent wires or sensors can be coupled via floating top gates. ${ }^{16}$ First experiments have been performed that demonstrate coupling of nanowire qubits to superconducting cavities, explored as universal on-chip quantum buses. ${ }^{17}$ For long-distance transfer of quantum information, a short section of a smaller bandgap semiconductor can be integrated in a nanowire of a larger bandgap material to produce an optically active quantum dot. ${ }^{18}$ With this quantum dot in a nanowire system, single photon emission and detection have recently been shown. ${ }^{19}$ In this article, we review recent progress made with small bandgap nanowires of InAs and InSb for quantum dot spin qubits and topological Majorana fermion qubits.

\section{Nanowire growth and electrical properties}

The state of the art in nanowire growth is based on the vapor-liquid-solid (VLS) mechanism, ${ }^{20}$ which can be used to fabricate a wide range of single-crystal semiconductors (Figure 1)..$^{21-29}$ Axial and radial heterostructures have been demonstrated, and dopants can be controllably introduced. ${ }^{21-24}$ For qubit devices, it is essential to have uniform carrier density and long mean free paths both to realize arrays of singleelectron quantum dots and to create welldefined topological phases in Majorana systems. Among the III-V semiconductors, InAs and InSb have the lowest effective electron mass, resulting in the highest electron mobili$\operatorname{ties}\left(\mu_{\mathrm{InAs}}=40,000 \mathrm{~cm}^{2} / \mathrm{V} \times \mathrm{s}\right.$ and $\mu_{\mathrm{InSb}}=77,000$ $\mathrm{cm}^{2} / \mathrm{V} \times \mathrm{s}$ at $300 \mathrm{~K}$ in the bulk $)^{30}$ and the largest confinement energies. For the qubits described here, large Landé g-factors, which define the energy a spin acquires in a magnetic field, and strong spin-orbit interaction in these materials are key, as described in subsequent sections. A minimum wire length of a few micrometers is needed to connect all electrodes, and the diameter should be below 100 nanometers to maintain electrostatic gate tunability.
VLS growth of arsenide and phosphide nanowires from gold catalyst particles is robust, ${ }^{24-26}$ but it remains challenging to reproducibly grow antimonides due to the lack of latticematched substrates, the low vapor pressure of $\mathrm{Sb}$, and its surfactant behavior. ${ }^{31-34}$ Because of its low surface energy, antimony has a tendency to float on top of the substrate and on the catalyst droplet without being incorporated. This makes it difficult to grow long and thin InSb nanowires. To initiate the growth of InSb nanowires in the $<111>$ B direction, InP-InAs stems are used as a starting point. The nanowire density on the substrate is controlled by defining arrays of catalyst particles using electron beam lithography. By optimizing the density and growth conditions, such as the temperature and III/V precursor ratio, it is possible to increase the aspect ratio (length/ diameter) of the nanowires up to 35 ; the longest $\mathrm{InSb}$ wires have lengths of up to $4 \mu \mathrm{m} .^{34}$

New ways to tailor electronic properties, such as crystal structure ${ }^{35}$ and strain, ${ }^{36}$ have recently been revealed. With nanowires, it is possible to fabricate common semiconductors with a different crystal structure than in bulk. For instance, phosphides and arsenides normally have a cubic structure,
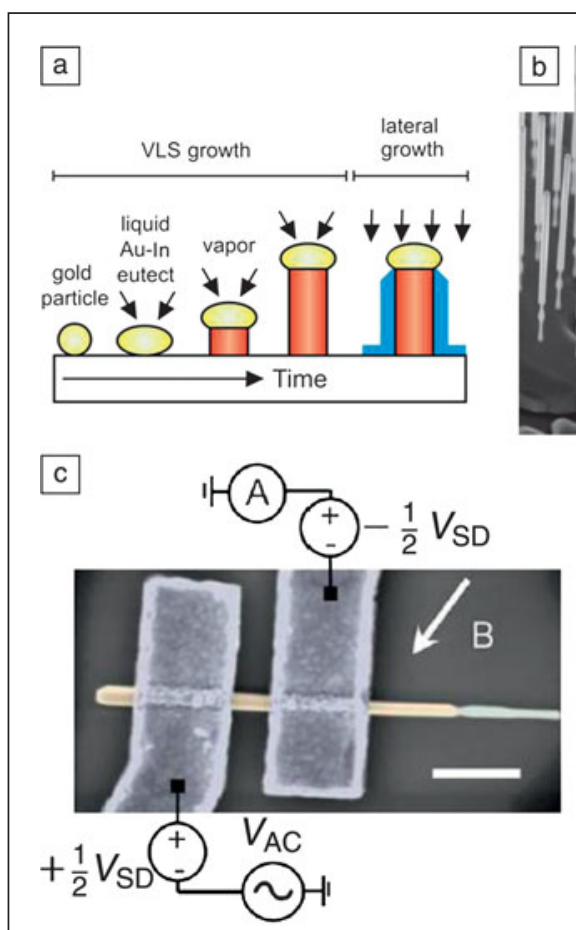
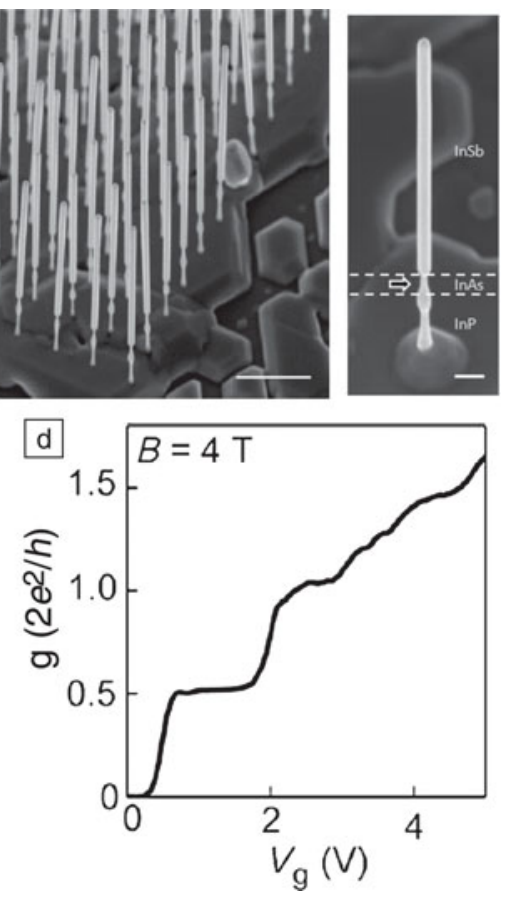

Figure 1. (a) Vapor-liquid-solid (VLS) nanowire growth mechanism. A metal catalyst particle collects precursor material from the vapor phase. The particles become liquid, and when saturated, a solid crystalline wire precipitates below the liquid catalyst. Growth in the vertical direction is promoted by the particle, but growth in the radial direction can also occur; this can be used to grow a shell of another material around the core wire. (b) Scanning electron microscope (SEM) image of InSb nanowires grown on an InP-InAs stem. These wires have a length of more than 3 microns and a diameter of $100 \mathrm{~nm}$. Scale bars correspond to 1 micron (left) and $200 \mathrm{~nm}$ (right). Reprinted with permission from Reference 34. (c) 2012 American Chemical Society. (c) SEM image of an InSb wire with two ohmic contacts. Arrow represents the orientation of the magnetic field. The scale bar is $500 \mathrm{~nm}$. The substrate is used as a global gate. (d) The conductance steps at $e^{2} / h$ demonstrate ballistic transport. Panels (c) and (d) are reprinted with permission from Reference 42. (c) 2013 American Chemical Society. 
but within a nanowire, the hexagonal structure is also formed. ${ }^{24-26,37}$ The band offset between different crystal structures has been used to create a new type of quantum structure: crystal phase dots. ${ }^{29}$ On the flip side, this presents a challenge in the form of uncontrolled variations of the crystal structure (polytypism) in nanowires, which is detrimental to electron transport. Indeed, theoretical predictions have indicated that zinc blende (ZB) and wurtzite (WZ) phases have different electronic band structures and stacking faults, and phase boundaries can act as scattering centers for electrons. ${ }^{38-40}$ For most III-V nanowires grown in the $<111>B$ direction, the crystalline structure is composed of a mixture of $\mathrm{ZB}$ and $\mathrm{WZ}$ phases. Perfect control of the InAs nanowire crystalline structure between $\mathrm{WZ}$ and $\mathrm{ZB}$ is nevertheless possible by optimizing growth conditions. ${ }^{25,26}$ InSb nanowires exhibit a pure ZB structure for a broad range of growth parameters. ${ }^{31-34}$

For small bandgap semiconductors, it is straightforward to fabricate low resistance electrical contacts by standard lithography and metal deposition. Low temperatures are required for semiconductor qubits in order to suppress thermal broadening of quantum levels below the typical level spacing of 1-10 meV. Nominally undoped InAs and InSb nanowires exhibit lowtemperature field-effect mobilities between 10,000 and 35,000 $\mathrm{cm}^{2} / \mathrm{V} \times \mathrm{s}$ with an electron concentration of $10^{17} \mathrm{~cm}^{3} \cdot{ }^{34,41}$ These mobilities are among the highest reported for nanowires, but they are lower than in the bulk or in remote doped 2D quantum wells. Nevertheless, ballistic transport has been reported through these nanowires. Ballistic transport manifests when the distance between the source and drain contacts is comparable to the electron mean free path in the nanowire, which is around $300 \mathrm{~nm}$ for InSb. ${ }^{42}$ Conductance exhibits quantized plateaus every $e^{2} / h$ (spin degeneracy is lifted by the magnetic field), meaning that electrons propagate through the nanowire as perfect waves in a waveguide. This is particularly challenging to demonstrate in nanowires, as even weak scattering will cause back-reflection of the electron wave and lead to the suppression of quantization in a one-dimensional system.

Possible causes for remaining carrier scattering are dangling bonds and adsorbates at the nanowire surface, or point defects, such as incorporated impurities (carbon atoms from precursors or gold atoms from the catalyst) or vacancies. Surface scattering may be reduced by growing a passivating shell around the wire. Incorporation of impurities can be avoided by using molecular beam epitaxy rather than metalorganic vapor phase epitaxy and employing a self-catalyzed growth mechanism as opposed to gold catalysts. Further progress in reducing defect density will lead to more robust conductance quantization over longer segments and enhance the robustness of quantum dot and Majorana states.

\section{Spin-based qubits in nanowires}

Electron spin is a two-level quantum system, which makes it a natural choice for a qubit. The ideal material for spin qubits must provide means for confining individual spins and for both coherent single spin rotations and controllable two-spin coupling. ${ }^{43}$ Importantly, the timescale at which spin orientation is lost (dephased) due to interactions with surrounding spins has to be many times longer than the time needed for a single quantum logic gate. ${ }^{1}$

Experiments on spin coherence in nanowires are performed in a double quantum dot configuration, where two single electrons are confined next to each other by five gate electrodes (Figure 2a). ${ }^{44}$ This, in principle, defines two qubits, but so far, nanowire experiments have focused on single spin states, while the second spin served as a detector. ${ }^{7}$ Detection relies on spin blockade of electron transport: If the two spins point in the same direction, tunneling of an electron from the left dot to the right dot is prohibited by the Pauli exclusion principle (Figure 2b). ${ }^{45,46}$ When the left electron is pushed to the right by a gate pulse, it can only shift if the two spins form a singlet state. This effect is also used for the initialization of the two qubits in the triplet state by pushing electrons out until a triplet is formed.

For the manipulation between spin-up and spin-down states, a standard technique is electron spin resonance. ${ }^{3}$ A highfrequency magnetic field is generated by a current in a micronscale coil fabricated near a quantum dot, and the duration of

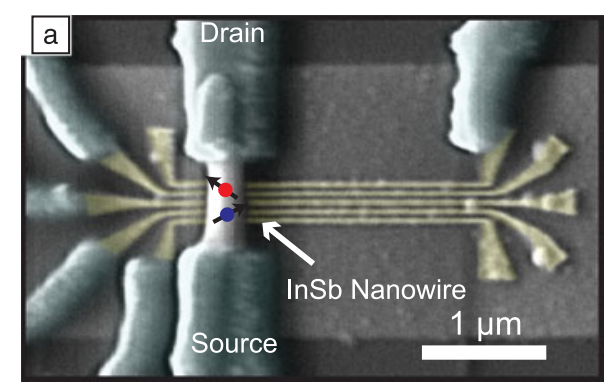

$\mathrm{b}$
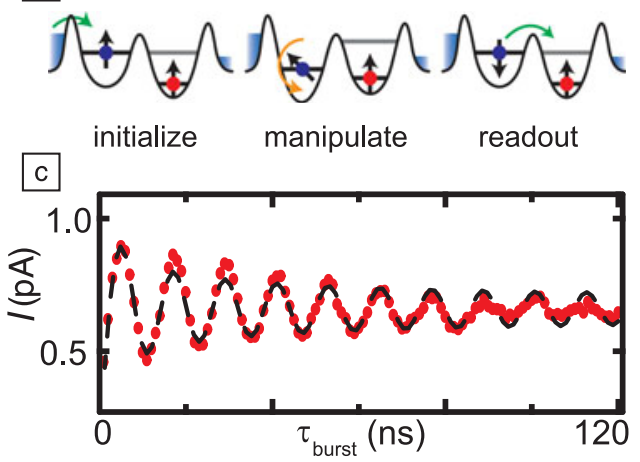

Figure 2. (a) Scanning electron microscope image of the nanowire double dot device. The electrons in the two dots (red and blue) are defined using five gate electrodes (colored in yellow). (b) Spin-blockade-based scheme for measuring spin rotations. In the first stage, the spin is initialized in the spinblocked state. In the manipulation stage, the dots are detuned, and bursts of electric fields are applied. Finally, in the readout stage, the spin blockade is used for detection of the final state. (c) An example of coherent Rabi oscillations. Reprinted with permission from Reference 48. () 2013 American Physical Society. 
the microwave pulse controls the spin rotation angle. This approach is not easily scalable, as coils tend to be large, and magnetic fields affect multiple nearby qubits. In small bandgap wires, spin resonance can be induced by local electric fields on the gate electrodes, a method that offers advantages for scalability. ${ }^{7,47}$ As the electron is moved back and forth by the gate, its spin rotates under the influence of the effective spin-orbit field. An experimental example of the coherent spin rotations induced by gigahertz electric fields in InSb nanowires is shown in Figure 2c.48

Due to the strong spin-orbit interaction (spin-orbit lengths are 100-200 $\mathrm{nm}$ for InAs and InSb), spin rotation frequencies beyond $100 \mathrm{MHz}$ can be achieved, the fastest among semiconductor-based single spin qubits. ${ }^{48}$ An important advantage of small bandgap semiconductor nanowires comes from the relatively large Landé g-factors (10 for InAs, 50 for InSb), meaning larger spin-up to spin-down energy splitting can be reached for small external constant magnetic fields. Furthermore, the g-factor in semiconductors is sensitive to the shape of a quantum dot, so electrons trapped in adjacent quantum dots have different g-factors. ${ }^{49}$ This feature turns out to be very useful for addressing individual qubits using different microwave tones.

A major limiting factor for all spin qubits based on III-V semiconductors is the short dephasing time. This is caused by nuclear spins that couple to the electron spin via hyperfine coupling. ${ }^{50}$ Techniques such as spin echo and dynamical decoupling can be used to partly cancel the influence of nuclear spins. ${ }^{2,51,52}$ In parallel, there are powerful ways to suppress nuclear spin fluctuations by electron-nuclear spin feedback. ${ }^{53-55}$ A completely different approach to overcome the nuclear spin problem is to use holes instead of electrons for qubits. ${ }^{56}$ Since holes occupy $p$ orbitals, hyperfine interaction is reduced by at least an order of magnitude. In InSb nanowires, it is possible to electrically tune the Fermi level between the conduction and valence bands and define a quantum dot with a single electron or a single hole in the same nanowire. ${ }^{57}$ On the way toward hole spin qubits, electrically driven spin resonance has been demonstrated with single holes in InSb nanowires.

Looking forward, it may be practical to use isotopes that do not have nuclear spins for obtaining longer electron spin coherence times. Such isotopes exist for the elements from even groups of the periodic table. Typical examples are wires made of silicon or germanium. Qubits made from these materials show extremely long spin coherence times. ${ }^{6,58}$ However, since the electron mass in silicon and germanium is quite large, it is more difficult to confine electrons. Also due to the large bandgap, careful engineering of the metallic contacts is needed in order to avoid the formation of Schottky barriers. A promising approach is to use hole spins in germanium since they have a low effective mass and demonstrate low resistance with conventional Ti/Au contacts. ${ }^{59-61}$ Ballistic transport, spin blockade, and single spin relaxation times have recently been measured in $\mathrm{Ge} / \mathrm{Si}$ core-shell nanowires on the way to a nuclear spinfree nanowire qubit. ${ }^{62}$ Strong spin-orbit interaction has been predicted in the valence band for $\mathrm{Ge} / \mathrm{Si}$ due to strain from the Si shell. ${ }^{63}$ This raises prospects for electrically induced spin control.

\section{Topological qubits and Majorana fermions}

Semiconductor nanowires have also gained prominence as a platform for the realization of a new paradigm of quantum computation based on topology. Decoherence induced by the qubit environment, the greatest challenge for all realizations of a quantum computer, can be sidestepped within topological quantum computing. ${ }^{64}$ The reason for this is that most decoherence boils down to a local perturbation acting on a qubit at its position. Topological qubits are prepared non-locally such that part of the quantum state is in point $\mathrm{A}$, and the other part is in point B far away. Small changes in the fields and forces at points $A$ and $B$ do not alter the qubit state. Only a radical operation of changing $\mathrm{A} \rightarrow \mathrm{B}$ and $\mathrm{B} \rightarrow \mathrm{A}$ transfers the qubit from one quantum state to the other. This immunity to local perturbations is called "topological protection"; it can be realized in solid-state systems with points A and B corresponding to the locations of exotic quasiparticles-Majorana fermions.

Majorana fermions were originally derived in the context of particle physics as real solutions to the Dirac equation, with neutrinos in mind.$^{65}$ For the purposes of topological qubits, the term "Majorana fermion" refers to quasiparticles that are their own antiparticles. To satisfy this, a single Majorana fermion must be an equal superposition of an electron and a hole. (Note that an exciton is a boson consisting of two fermionsan electron and a hole, while a Majorana fermion is "a half of an electron plus a half of a hole"). Superpositions of electrons and holes commonly occur in superconductors, with electrons being excitations above the Fermi level, and holes being empty states below the Fermi level. But in most conventional superconductors, electron-hole superpositions are rapidly destroyed due to quantum fluctuations. For topological qubits, it is necessary to create Majorana fermions that are robust in time. To this end, a number of exotic materials in which quantum fluctuations are canceled out by the band topology were proposed, ranging from $p$-wave superconductors to fractional Quantum Hall edge states and topological insulators. ${ }^{66-68}$

Recently, a recipe has been put forward for how to generate a pair of spatially separated Majorana fermions in nanowire-based devices. ${ }^{69-71}$ This proposal is relatively straightforward because it contains just four common ingredients: (1) a one-dimensional electronic system with (2) spinorbit interaction and (3) superconductivity in (4) a magnetic field. The magnetic field lifts spin degeneracy, while spinorbit interaction mixes spin-up and spin-down and through that ensures that superconductivity based on coupling electrons with opposite spins is still preserved. The one-dimensional template ensures that only two Majorana fermions are created, one at each end of the nanowire. Longer nanowires offer better topological protection.

The experimental implementation of this recipe has focused on InSb nanowires. ${ }^{34}$ Strong spin-orbit interaction has already 
been established in this material in spin-orbit qubit experiments. ${ }^{72,73}$ The highest electron mobility among nanowires was measured in InSb nanowires; this offered another advantage, as disorder could lead to the creation of additional Majorana pairs along the nanowire due to fluctuations in the chemical potential. Furthermore, Schottky barriers at the metal/ nanowire junction are very small in InSb, which facilitates high-transparency contacts to superconductors. ${ }^{74}$ Finally, large electronic Landé g-factors in InSb mean that small magnetic fields can be sufficient for generating Majorana fermions.

Devices fabricated for the Majorana experiment consisted of an InSb nanowire contacted by two electrodes, a superconducting $\mathrm{NbTiN}$, and a Ti/Au non-superconducting contact (Figure 3). ${ }^{8}$ The superconducting contact induces superconductivity in the semiconductor by proximity, as required by the Majorana recipe. The purpose of the gold contact is to detect the Majorana quasiparticle. Underneath the nanowire, an array of electrostatic gate electrodes is fabricated in order to locally tune the density of charges in the semiconductor, similar to spin qubits. One narrow gate between the two contacts is set to a negative voltage in order to induce a tunneling barrier in the nanowire. A Majorana state is expected to appear at the barrier on the superconducting side. The non-superconducting
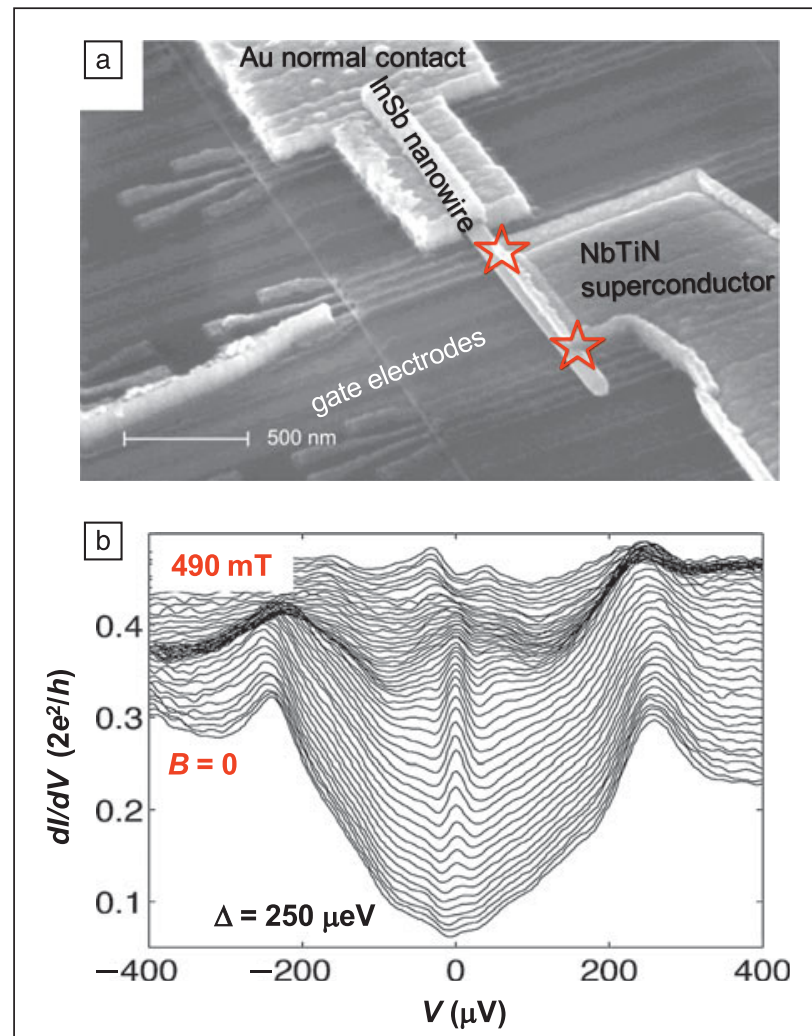

Figure 3. (a) Scanning electron microscope image of a Majorana experiment device. Red stars indicate positions where Majorana fermions are expected. (b) Experimental data showing the zero-bias peak as it appears at a magnetic field of $\sim 100 \mathrm{mT}$. Curves are taken every $10 \mathrm{mT}$ and are offset for clarity. Reprinted with permission from Reference 8. (C) 2012 American Association for the Advancement of Science. side of the wire is used as a tunneling probe of the Majorana quasiparticle, analogous to a scanning tunneling microscope tip but within the nanowire itself.

Because a Majorana fermion is an equal superposition of an electron and a hole, it must appear at the boundary between electrons and holes, at the Fermi level in a superconductor. And since it is a localized quantum state, it is predicted to produce a peak in the tunneling current, at zero applied voltage bias between Ti/Au and NbTiN electrodes. Such zero-bias peaks were indeed observed in conductance. The peaks appeared at finite field as prescribed by the Majorana recipe. Additional tests for the Majorana nature of the zero-bias peaks included their stability over a range of magnetic field and gate voltages underneath the superconductor, properties consistent with topologically robust states. The relevance of spin-orbit interaction was confirmed by observing that zero-bias peaks vanish when the magnetic field is aligned with the spin-orbit field, leading to the annihilation of Majorana fermions. Several other experiments in semiconductor nanowire-superconductor hybrids recently reported zero-bias peaks, though for those studies, explanations such as the Kondo effect, Josephson effect, and Andreev bound states are hard to rule out in the absence of more detailed studies of the zero-bias peak sensitivity of system parameters, as discussed previously. ${ }^{75-77}$

To date, the most exotic property of Majorana fermions that makes them attractive for topological qubits has not yet been observed. The non-Abelian character of these quasiparticles can be understood in the following way: ${ }^{78}$ When two identical particles exchange their positions, the universe remains unchanged. This indistinguishability principle is absolute for all known bosons and fermions, but not for Majoranas, which makes them not quite fermions. When two Majoranas exchange positions, the system transitions from one quantum ground state to another distinct state. In nanowires, the two states can be different by a charge $e$ (i.e., two Majoranas can be thought of as a box for a single electron that can be either full or empty). The two states of the Majorana box represent the two states of a topological qubit. The operation of Majorana interchange in space is known as "braiding."

\section{Outlook}

In this article, we have only discussed homogeneous wires, but various materials can be combined into a single wire during the growth process. In such wires, different parts can be used for different purposes. For example, one can perform fast spin manipulation in a segment of the wire made of a III-V material and store the information (i.e., the electron spin in another part made of silicon where nuclear spins do not cause decoherence). Recently, Si wires with GaAs segments have been demonstrated. ${ }^{14}$ This should be expanded toward combinations with smaller bandgap III-Vs, such as InSb, but then the lattice mismatch between these materials may become problematic. This is an open challenge in materials science. Another option would be to combine InSb with II-VI semiconductors, such as CdTe, which are almost lattice matched 
and have isotopes without nuclear spin. These exotic material combinations have been shown in $2 \mathrm{DEGs}^{79}$ but not yet in nanowires.

For topological qubits, a major materials challenge is that braiding is not possible in a single nanowire. Indeed, to exchange two Majorana fermions located at the edges of a nanowire, one needs to move them along the wire (e.g., by locally changing the chemical potential on the gates). At one point, they will meet and annihilate, which will lead to a collapse of the qubit state. Nanowire structures with an extra leg, so called T-shapes, are required to perform braiding. ${ }^{80}$ One Majorana fermion can be located in the third leg, while the second Majorana fermion is moved across. A materials challenge is to fabricate high-quality T-shaped wires with all the requirements mentioned previously. ${ }^{81}$ As nanowire growth methods develop, new possibilities open up for qubits with improved characteristics as well as for fundamentally new quantum devices.

\section{References}

1. R. Hanson, L.P. Kouwenhoven, J.R. Petta, S. Tarucha, L.M.K. Vandersypen, Rev. Mod. Phys. 79 (4), 1217 (2007).

2. J.R. Petta, A.C. Johnson, J.M. Taylor, E.A. Laird, A. Yacoby, M.D. Lukin, C.M. Marcus, M.P. Hanson, A.C. Gossard, Science 309 (5744), 2180 (2005)

3. F.H.L. Koppens, C. Buizert, K.J. Tielrooij, I.T. Vink, K.C. Nowack, T. Meunier, L.P. Kouwenhoven, L.M.K. Vandersypen, Nature 442 (7104), 766 (2006)

4. K.D. Petersson, J.R. Petta, H. Lu, A.C. Gossard, Phys. Rev. Lett. 105 (24), 246804 (2010).

5. M.D. Shulman, O.E. Dial, S.P. Harvey, H. Bluhm, V. Umansky, A. Yacoby, Science 336 (6078), 202 (2012)

6. B.M. Maune, M.G. Borselli, B. Huang, T.D. Ladd, P.W. Deelman, K.S. Holabird, A.A. Kiselev, I. Alvarado-Rodriguez, R.S. Ross, A.E. Schmitz, M. Sokolich, C.A. Watson, M.F. Gyure, A.T. Hunter, Nature 481 (7381), 344 (2012).

7. S. Nadj-Perge, S.M. Frolov, E.P.A.M. Bakkers, L.P. Kouwenhoven, Nature 468 (7327), 1084 (2010).

8. V. Mourik, K. Zuo, S.M. Frolov, S.R. Plissard, E.P.A.M. Bakkers, L.P. Kouwenhoven, Science 336 (6084), 1003 (2012).

9. Y.Y. Wu, R. Fan, P.D. Yang, Nano Lett. 2 (2), 83 (2002)

10. M.T. Björk, B.J. Ohlsson, T. Sass, A.I. Persson, C. Thelander, M.H. Magnusson, K. Deppert, L.R. Wallenberg, L. Samuelson, Nano Lett. 2 (2), 87 (2002).

11. M.T. Borgström, V. Zwiller, E. Müller, A. Imamoglu, Nano Lett. 5 (7), 1439 (2005).

12. J. Ristić, E. Calleja, M.A. Sánchez-Garcia, J.M. Ulloa, J. Sánchez-Páramo, J.M. Calleja, U. Jahn, A. Trampert, K.H. Ploog, Phys. Rev. B 68 (12), 125305 (2003).

13. A. Tribu, G. Sallen, T. Aichele, R. André, J.P. Poizat, C. Bougerol, S. Tatarenko, K. Kheng, Nano Lett. 8 (12), 4326 (2008).

14. M. Hocevar, G. Immink, M. Verheijen, N. Akopian, V. Zwiller, L.P. Kouwenhoven, E.P.A.M. Bakkers, Nat. Commun. 3, 1266 (2012).

15. C. Fasth, A. Fuhrer, M.T. Björk, L. Samuelson, Nano Lett. 5 (7), 1487 (2005). 16. Y.J. Hu, H.O.H. Churchill, D.J. Reilly, J. Xiang, C.M. Lieber, C.M. Marcus, Nat. Nanotechnol. 2 (10), 622 (2007).

17. K.D. Petersson, L.W. McFaul, M.D. Schroer, M. Jung, J.M. Taylor, A.A. Houck, J.R. Petta, Nature 490 (7420), 380 (2012).

18. E.D. Minot, F. Kelkensberg, M. van Kouwen, J.A. van Dam, L.P. Kouwenhoven, V. Zwiller, M.T. Borgström, O. Wunnicke, M.A. Verheijen, E.P.A.M. Bakkers, Nano Lett. 7 (2), 367 (2007).

19. M.E. Reimer, G. Bulgarini, H. Akopian, M. Hocevar, M.B. Bavinck, M.A. Verheijen, E.P.A.M. Bakkers, L.P. Kouwenhoven, V. Zwiller, Nat. Commun. 3, 737 (2012).

20. R.S. Wagner, W.C. Ellis, Appl. Phys. Lett. 4 (5), 89 (1964).

21. M.S. Gudiksen, L.J. Lauhon, J. Wang, D.C. Smith, C.M. Lieber, Nature 415 (6872), 617 (2002)

22. T. Kuykendall, P. Ulrich, S. Aloni, P. Yang, Nat. Mater. 6 (12), 951 (2007).

23. M.T. Björk, C. Thelander, A.E. Hansen, L.E. Jensen, M.W. Larsson, L.R. Wallenberg, L. Samuelson, Nano Lett. 4 (9), 1621 (2004).

24. R.E. Algra, M.A. Verheijen, M.T. Borgstrom, L.F. Feiner, G. Immink, W.J.P. van Enckevort, E. Vlieg, E.P.A.M. Bakkers, Nature 456 (7220), 369 (2008). 25. P. Caroff, K.A. Dick, J. Johansson, M.E. Messing, K. Deppert, L. Samuelson, Nat. Nanotechnol. 4 (1), 50 (2009).

26. H.J. Joyce, J. Wong-Leung, Q. Gao, H.H. Tan, C. Jagadish, Nano Lett. 10 (3), 908 (2010).
27. A. Fontcuberta i Morral, J. Arbiol, J.D. Prades, A. Cirera, J.R. Morante, Adv. Mater. 19 (10), 1347 (2007).

28. D. Spirkoska, J. Arbiol, A. Gustafsson, S. Conesa-Boj, F. Glas, I. Zardo, M. Heigoldt, M.H. Gass, A.L. Bleloch, S. Estrade, M. Kaniber, J. Rossler, F. Peiro, J.R. Morante, G. Abstreiter, L. Samuelson, A. Fontcuberta i Morral, Phys. Rev. B 80 (24), 245325 (2009).

29. N. Akopian, G. Patriarche, L. Liu, J.C. Harmand, V. Zwiller, Nano Lett. 10 (4), 1198 (2010).

30. www.ioffe.rssi.ru/SVA/NSM/Semicond

31. P. Caroff, M.E. Messing, B.M. Borg, K.A. Dick, K. Deppert, L.E. Wernersson, Nanotechnology 20, 495606 (2009).

32. L. Lugani, D. Ercolani, F. Rossi, G. Salviati, F. Beltram, L. Sorba, Cryst. Growth Des. 10 (9), 4038 (2010).

33. A.T. Vogel, J. de Boor, J.V. Wittemann, S.L. Mensah, P. Werner, V. Schmidt, Cryst. Growth Des. 11 (5), 1896 (2011).

34. S.R. Plissard, D.R. Slapak, M.A. Verheijen, M. Hocevar, G.W.G. Immink, I. van Weperen, S. Nadj-Perge, S.M. Frolov, L.P. Kouwenhoven, E.P.A.M. Bakkers, Nano Lett. 12 (4), 1794 (2012).

35. S. Assali, I. Zardo, S. Plissard, D. Kriegner, M.A. Verheijen, G. Bauer, A. Meijerink, A. Belabbes, F. Bechstedt, J.E.M. Haverkort, E.P.A.M. Bakkers, Nano Lett. 13 (4), 1559 (2013).

36. R.A. Minamisawa, M.J. Suess, R. Spolenak, J. Faist, C. David, J. Gobrecht, K.K. Bourdelle, H. Sigg, Nat. Commun. 3, 1096 (2012).

37. H. Shtrikman, R. Popovitz-Biro, A. Kretinin, L. Houben, M. Heiblum, M. Bukala, M. Galicka, R. Buczko, P. Kacman, Nano Lett. 9 (4), 1506 (2009).

38. C.Y. Yeh, S.H. Wei, A. Zunger, Phys. Rev. B 50 (4), 2715 (1994).

39. A. De, C.E. Pryor, Phys. Rev. B 81 (15), 155210 (2010).

40. A. Belabbes, C. Panse, J. Furthmüller, F. Bechstedt, Phys. Rev. B 86 (7), 075208 (2012)

41. J.W.W. van Tilburg, R.E. Algra, W.G.G. Immink, M. Verheijen, E.P.A.M. Bakkers, L.P. Kouwenhoven, Semicond. Sci. Technol. 25 (2), 024011 (2010).

42. I. van Weperen, S.R. Plissard, E.P.A.M. Bakkers, S.M. Frolov, L.P. Kouwenhoven, Nano Lett. 13 (2), 387 (2013).

43. D. Loss, D.P. DiVincenzo, Phys. Rev. A 57 (1), 120 (1998).

44. S. Nadj-Perge, S.M. Frolov, J.W.W. van Tilburg, J. Danon, Y.V. Nazarov, R. Algra, E.P.A.M. Bakkers, L.P. Kouwenhoven, Phys. Rev. B 81 (20), 201305 (2010).

45. K. Ono, D.G. Austing, Y. Tokura, S. Tarucha, Science 297 (5585), 1313 (2002) 46. A. Pfund, I. Shorubalko, K. Ensslin, R. Leturcq, Phys. Rev. Lett. 99 (3) 036801 (2007)

47. K.C. Nowack, F.H.L. Koppens, Y.V. Nazarov, L.M.K. Vandersypen, Science 318 (5855), 1430 (2007).

48. J.W.G. van den Berg, S. Nadj-Perge, V.S. Pribiag, S.R. Plissard, E.P.A.M. Bakkers, S.M. Frolov, L.P. Kouwenhoven, Phys. Rev. Lett. 110 (6), 066806 (2013).

49. M.D. Schroer, K.D. Petersson, M. Jung, J.R. Petta, Phys. Rev. Lett. 107 (17), 176811 (2011).

50. F.H.L. Koppens, J.A. Folk, J.M. Elzerman, R. Hanson, L.H.W. van Beveren, I.T. Vink, H.P. Tranitz, W. Wegscheider, L.P. Kouwenhoven, L.M.K. Vandersypen, Science 309 (5739), 1346 (2005).

51. F.H.L. Koppens, K.C. Nowack, L.M.K. Vandersypen, Phys. Rev. Lett. 100 (23), 236802 (2008)

52. H. Bluhm, S. Foletti, I. Neder, M. Rudner, D. Mahalu, V. Umansky, A. Yacoby, Nat. Phys. 7 (2), 109 (2011).

53. I.T. Vink, K.C. Nowack, F.H.L. Koppens, J. Danon, Y.V. Nazarov, L.M.K. Vandersypen, Nat. Phys. 5 (10), 764 (2009).

54. S. Foletti, H. Bluhm, D. Mahalu, V. Umansky, A. Yacoby, Nat. Phys. 5 (12), 903 (2009).

55. S.M. Frolov, J. Danon, S. Nadj-Perge, K. Zuo, J.W.W. van Tilburg, V.S. Pribiag, J.W.G. van den Berg, E.P.A.M. Bakkers, L.P. Kouwenhoven, Phys. Rev. Lett. 109 (23), 236805 (2012)

56. K. De Greve, P.L. McMahon, D. Press, T.D. Ladd, D. Bisping, C. Schneider, M. Kamp, L. Worschech, S. Höfling, A. Forchel, Y. Yamamoto, Nat. Phys. 7 (11), 872 (2011).

57. V.S. Pribiag, S. Nadj-Perge, S.M. Frolov, J.W.G. van den Berg, I. van Weperen, S.R. Plissard, E.P.A.M. Bakkers, L.P. Kouwenhoven, Nat. Nanotechnol. 8 (3), 170 (2013).

58. A. Morello, J.J. Pla, F.A. Zwanenburg, K.W. Chan, K.Y. Tan, H. Huebl, M. Möttönen, C.D. Nugroho, C. Yang, J.A. van Donkelaar, A.D.C. Alves, D.N. Jamieson, C.C. Escott, L.C.L. Hollenberg, R.G. Clark, A.S. Dzurak, Nature 467 (7316), $687(2010)$

59. J. Xiang, A. Vidan, M. Tinkham, R.M. Westervelt, C.M. Lieber, Nat. Nanotechnol. 1 (3), 208 (2006).

60. J. Xiang, W. Lu, Y.J. Hu, Y. Wu, H. Yan, C.M. Lieber, Nature 441 (7092), 489 (2006).

61. W. Lu, J. Xiang, B.P. Timko, Y. Wu, C.M. Lieber, Proc. Natl. Acad. Sci. U.S.A. 102 (29), 10046 (2005).

62. Y.J. Hu, F. Kuemmeth, C.M. Lieber, C.M. Marcus, Nat. Nanotechnol. 7 (1), 47 (2012). 
63. C. Kloeffel, M. Trif, D. Loss, Phys. Rev. B 84 (19), 195314 (2011).

64. A. Stern, N.H. Lindner, Science 339 (6124), 1179 (2013).

65. F. Wilczek, Nat. Phys. 5 (9), 614 (2009).

66. C. Nayak, S.H. Simon, A. Stern, M. Freedman, S. Das Sarma, Rev. Mod. Phys. 80 (3), 1083 (2008).

67. D.A. Ivanov, Phys. Rev. Lett. 86 (2), 268 (2001).

68. L. Fu, C.L. Kane, Phys. Rev. Lett. 100 (9), 096407 (2008).

69. A.Y. Kitaev, Phys. Usp. 44, 131 (2001).

70. R.M. Lutchyn, J.D. Sau, S. Das Sarma, Phys. Rev. Lett. 105 (7), 077001 (2010).

71. Y. Oreg, G. Refael, F. von Oppen, Phys. Rev. Lett. 105 (17), 177002 (2010).

72. S. Nadj-Perge, V.S. Pribiag, J.W.G. van den Berg, K. Zuo, S.R. Plissard, E.P.A.M. Bakkers, S.M. Frolov, L.P. Kouwenhoven, Phys. Rev. Lett. 108 (16), 166801 (2012).

73. H.A. Nilsson, P. Caroff, C. Thelander, M. Larsson, J.B. Wagner, L.E. Wernersson, L. Samuelson, H.Q. Xu, Nano Lett. 9 (9), 3151 (2009).
74. H.A. Nilsson, P. Samuelsson, P. Caroff, H.Q. Xu, Nano Lett. 12 (1), 228 (2012).

75. M.T. Deng, C.L. Yu, G.Y. Huang, M. Larsson, P. Caroff, H.Q. Xu, Nano Lett. 12 (12), 6414 (2012)

76. A. Das, Y. Ronen, Y. Most, Y. Oreg, M. Heiblum, H. Shtrikman, Nat. Phys. 8 (12), 887 (2012)

77. A.D.K. Finck, D.J. Van Harlingen, P.K. Mohseni, K. Jung, X. Li, Phys. Rev. Lett. 110 (12), 126406 (2013).

78. N. Read, D. Green, Phys. Rev. B 61 (15), 10267 (2000).

79. S.K. Greene, J. Singleton, P. Sobkowicz, T.D. Golding, M. Pepper, J.A.A.J. Perenboom, J. Dinan, Semicond. Sci. Technol. 7 (11), 1377 (1992).

80. J. Alicea, Y. Oreg, G. Refael, F. von Oppen, M.P.A. Fisher, Nat. Phys. 7 (5), 412 (2011).

81. D. Dalacu, A. Kam, D.G. Austing, P.J. Poole, Nano Lett. 13 (6), 2676 (2013).

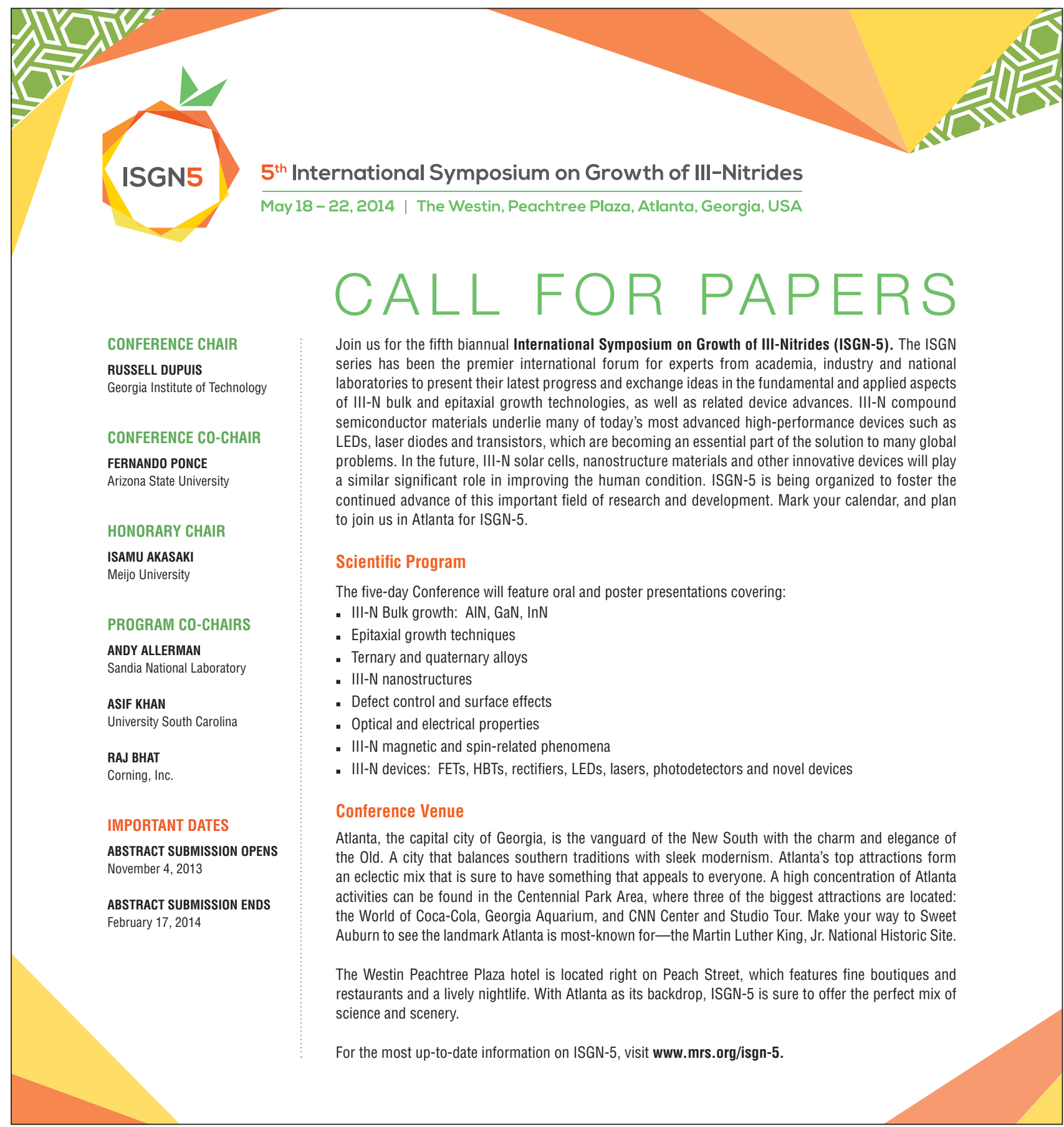

